\title{
A Deep Survey for Faint Galactic Wolf-Rayet Stars
}

\author{
M. M. Shara ${ }^{1}$, M. Potter ${ }^{1}$, A. F. J. Moffat ${ }^{2}$, L. F. Smith ${ }^{3}$ \\ ${ }^{1}$ Space Telescope Science Institute, 3700 San Martin Drive, Baltimore, MD 21218 \\ ${ }^{2}$ University of Montreal, Dept. of Physics, C.P. 6128, Succ. A., Montreal PQ H3C 3J7, Canada \\ ${ }^{3}$ Mount Stromlo and Siding Springs Observatory, Private Bag, P.O. Woden ACT 2606, Australia
}

\begin{abstract}
Surveys of the Galaxy for Wolf-Rayet (WR) stars are mostly based on objective prism searches, and are generally complete to only about 13 th visual magnitude. We are using direct narrowband and broadband Schmidt plates to survey large areas of the southern Milky Way for WR stars to 17-18th magnitude. We expect to find more than 50 new WR stars. The newly detected stars should be among the most distant and/or reddened known in the Galaxy. The survey is also designed to test the completeness of previous bright WR star surveys, and thus to help settle debates over the Initial Mass Function of the most massive stars. We have now located 13 new WR stars in a 40 square degree region in Carina where 24 WR stars were already known. A $25 \%$ incompleteness in detection of WR stars as close as 2-3 kpc is suggested.
\end{abstract}

\section{Wolf-Rayet Stars Surveys}

Narrowband images taken with CCDs and 4 meter telescopes can reveal 24th magnitude Wolf-Rayet stars at the distance of the M81 group of galaxies; however, our own Galaxy has been completely surveyed only to $m \simeq 13-14$ for WR stars (based on the latest Galactic WR catalogue; van der Hucht et al. 1981, updated by van der Hucht et al. 1988). Only 12 of 157 known Galactic WR stars are fainter than $V=15$. This is indicative of the crowding problems which limit objective prism surveys in the Galactic plane.

\section{Motivation for the Present WR Star Survey}

Benefits of the survey are to search a much larger part of the Galaxy for massive star formation (WC and WN stars) than has hitherto been accomplished; to verify and extend the result that the ratio of WC to WN stars varies with Galactocentric distance; and to locate very distant WR stars to act as probes of the interstellar medium, in the UV, visible and IR, in spiral arms far from the Sun.

\section{Narrowband-Broadband Imaging and Spectroscopic Followup}

By virtue of their generally strong, broad emission lines, WR stars can be easily detected at faint magnitudes in extended regions by comparing direct images taken with narrow on-versus broad on-line interference filters. WR stars appear considerably brighter (0.2-0.7 
magnitudes) in the narrowband images than non-WR stars. All plates are digitized, and all HeII-bright candidates are detected in objective fashion via computer analysis of the images.

With this technique (unlike objective prism plates) confusion is not a problem even in the Galactic plane at 18-19th magnitude. These filters successfully detect weak-lined WN stars in very crowded fields (Moffat, Seggewiss and Shara 1985). We note that two of the new objects are members of clusters and a third is only 3" from a previously known WR star. Thus our search technique is particularly useful at finding WR stars in crowded fields missed by previous, confusion-limited objective prism surveys.

Spectroscopic checking of every candidate which passes all the photometric tests must be carried out. This is to ensure that the candidates really are WR stars, and to obtain their subtypes.

\section{Results}

\subsection{Galactic Distribution}

The previously noted (Conti and Vacca 1990) tendency for WR stars to be found below the Galactic equator is strongly reinforced. All 13 new WR stars lie at $b<0^{\circ}$, which may be indicative of a warp in the Galactic disk.

The 13 new WR stars are significantly fainter and redder than the previously known Carina stars. This increased reddening is, of course, most readily interpreted as being due to increased distance from the Sun. The extension of the Carina arm is more striking than ever. Three of the thirteen new stars are closer than $3 \mathrm{kpc}$., in comparison with 9 known stars within the same distance. This sugguests an incompleteness in the present WR census of $\sim 25 \%$ even for the closeby stars.

While individual stars' Galactocentric distances $R$ are somewhat uncertain, the overall trend is not. The average $R$ of the 13 new stars is 11 or $11.7 \mathrm{kpc}$ (from the van der Hucht et al. 1988 and Conti and Vacca 1990 calibrations, respectively). This is further out from the Galactic center than all but eight of the known Galactic WR stars. Thus our new stars are an extremely useful sample for testing whether the predicted and observed trends amoung WR stars in galaxies are confirmed in the outer Milky Way. Probably the most important of these is the decreasing WC/WN number ratio at increasing galactocentric distances.

\subsection{Subtypes}

One of the most striking results of this survey so far is the remarkable ratio of WC to WN stars found: 2 to $11(0.18)$, respectively. Conti and Vacca (1990) noted that the WC/WN ratio is 0.95 inwards of the solar circle $(8.5 \mathrm{kpc})$ and 0.74 outside. Smith's (1988) study demonstrates the WC/WNE dependence on $\log [\mathrm{O} / \mathrm{H}]$ (and hence Galactocentric distance). The dramatic decrease in WC/WN number ratio continues the trend seen in her Figure 2.

The low WC/WN ratio seen in our sample is also supportive of the work of Maeder (1990). His models predict that initial mass and metallicity together determine the lifetimes of massive stars in the WR phase. Thus at increasing $R$ the decreasing metallicity and smaller numbers of very massive stars conspire to strongly decrease the WC/WN ratio, as we now observe.

Future papers in this series will extend our search over much of the Southern Milky Way, and will concentrate on the global distribution of WR stars in the Galaxy. 


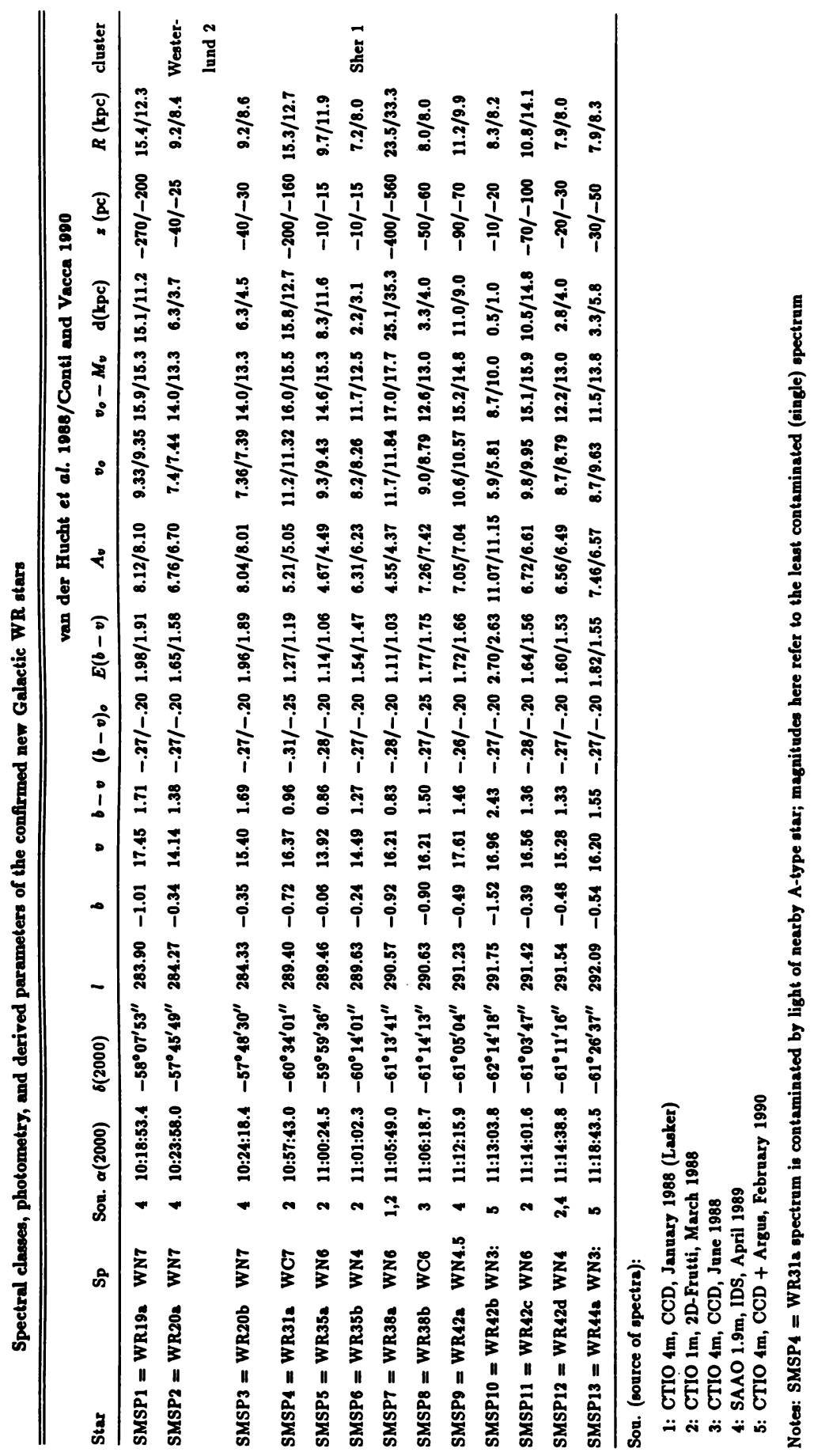




\section{References}

Conti, P.S., Vacca, W.D. 1990, Astron. J. 100, 431.

Maeder, A. 1990, Astron. Astrophys., in press.

Moffat, A.F.J., Seggewiss, W., Shara, M.M. 1985, Astrophys. J. 295, 109.

Smith, L.F. 1988, Astrophys. J. 327, 128.

van der Hucht, K.A., Conti, P.S., Lunström, I., Stenholm, B. 1981, Space Science Reviews 28, 227. van der Hucht, K.A., Hidayat, B., Admiranto, A.G., Supelli, K.R., Doom, C. 1988, Astron. Astro-

phys. 199, 217.

\section{DISCUSSION}

Montmerle: In view of the large incompleteness your work shows to exist in previous census, would you exclude the possibility that the total number of WR stars in our galaxy could be higher by factors 2-3, say, than the $\sim 2500$ quoted by van der Hucht et al. (1988)? This would be important for estimating the high-energy diffuse flux (galactic X-ray ridge, nuclear $\gamma$-ray line emission) that has been associated with these stars (Montmerle, this volume), particularly in the inner galaxy.

Shara: If you are talking about the entire galaxy there are numbers that are bandied about of a 1000 of even higher, and I do not think I would find that terribly shocking. There are certainly stars, very far away, that we have found here, but we may be in an unfair advantage, in a sense, by looking along the Carina arm. We may have less reddening, e.g., than in the other regions. I do not think that our results tell us how many WR stars there are in the galaxy. They are only telling us that the present survey is moderately complete down to the fourteenth magnitude. It is really a magnitude limit that is sampled rather than a volume limit. But even at fourteenth magnitude, there are stars waiting to be discovered. Thus, it is certainly possible, but we must expand the survey significantly to make an educated statement.

Massey: "Gradients" often imply two points, rather than one - do you not need to look in another direction with your sensitive technique before knowing if you have found a WC/WN gradient in the Galaxy?

Shara: Absolutely! I was simply taking the WC/WN number ratio close to the Sun $(\sim 1)$ as deduced from the Sixth Catalogue, and comparing with the $2 / 11=1.18$ ratio we find in our survey. Perhaps we see a strong gradient, as our new WR stars are at an average galactocentric distance of $11.4 \mathrm{kpc}$. But, perhaps we are just more complete than all previous surveys for WN stars, and we may find a small WC/WN ratio even when we look inwards in the Galaxy.

Conti: Speaking from a purely personal point of view, I would not be surprised if the total estimate of the number of WR stars galaxy-wide is at least a factor two too low. A factor ten is not impossible. Discovery of additional WR stars is completely dominated by the extinction problem.

van der Hucht: I must compliment you on this virtual monk's work, and I do hope to see your Seventh galactic WR Catalogue in my lifetime. I can understand that it is not always easy to keep up the good spirit during all this hard work. Therefore, I can understand that you once, in a sad mood, confessed to Tony Moffat: "Roses are red, carnations are pink. If I had your talents, I didn't have to blink." But please: carry on!

Shara: I promise to continue if you promise not to publish any more poetry.

Hidayat: Would you undertake surveys in the direction of low-absorption galactic fields (such as Puppis, at $l \sim 240^{\circ}$ )?

Shara: We will first do $l=282^{\circ}$ to $l=332^{\circ}$. If we succeed in completing this, then we will consider increased coverage. We must measure magnitudes and positions of $4 \times 10^{7}$ objects to complete the present survey. 